ANNALES

UNIVERSITATIS MARIAE CURIE-SKŁODOWSKA

LUBLIN - POLONIA

VOL. LXII, 2008

SECTIO A

$61-65$

K. K. DEWAN and SUNIL HANS

\title{
Growth of polynomials whose zeros are outside a circle
}

\begin{abstract}
If $p(z)$ be a polynomial of degree $n$, which does not vanish in $|z|<k, k<1$, then it was conjectured by Aziz [Bull. Austral. Math. Soc. 35 (1987), 245-256] that

$$
\max _{|z|=r}|p(z)| \geq \frac{r^{n}+k^{n}}{1+k^{n}} \max _{|z|=1}|p(z)| \text { for } k^{2}<r<1 .
$$

In this paper, we consider the case $k<r<1$ and present a generalization as well as improvement of the above inequality.
\end{abstract}

1. Introduction and statement of results. Let $p(z)$ be a polynomial of degree $n$ and let $M(p, R)=\max _{|z|=R}|p(z)|$. Then it is a simple consequence of maximum modulus principle (for reference see [4, vol. I, p. 137, prob. III, 269] that

$$
M(p, R) \leq R^{n} M(p, 1) \text { for } R \geq 1 .
$$

The result is best possible and equality holds for $p(z)=\alpha z^{n}$, where $|\alpha|=1$.

2000 Mathematics Subject Classification. 30A10, 30C10, 30E15.

Key words and phrases. Polynomials, inequalities, restricted zeros, growth.

The work is supported by Council of Scientific and Industrial Research, New Delhi, under grant F.No. 9/466(95)/2007-EMR-I. 
It was shown by Ankeny and Rivlin [1] that if $p(z) \neq 0$ in $|z|<1$, then inequality (1.1) can be replaced by

$$
M(p, R) \leq \frac{R^{n}+1}{2} M(p, 1) \text { for } R \geq 1 .
$$

The above inequality is best possible and equality holds for $p(z)=\alpha+\beta z^{n}$, where $|\alpha|=|\beta|$.

As a generalization of inequality (1.2), Aziz [2] conjectured the following results.

Conjectured results. If $p(z)$ is a polynomial of degree $n$, which does not vanish in $|z|<k$, then

$$
M(p, r) \geq \frac{r^{n}+k^{n}}{1+k^{n}} M(p, 1) \text { for } k^{2}<r<1, k<1
$$

and

$$
M(p, R) \leq \frac{R^{n}+k^{n}}{1+k^{n}} M(p, 1) \text { for } R>k^{2}, k>1 .
$$

In the same paper, Aziz [2] was able to prove inequality (1.4).

In an attempt to answer the inequality (1.3) conjectured by Aziz, we have been able to prove the following result.

Theorem 1. If $p(z)$ is a polynomial of degree $n$, which does not vanish in $|z|<k, k<1$, then for $0<k<r<\lambda \leq 1$

$$
M(p, r) \geq \frac{r^{n}+k^{n}}{\lambda^{n}+k^{n}} M(p, \lambda),
$$

provided $\left|p^{\prime}(z)\right|$ and $\left|q^{\prime}(z)\right|$ attain the maximum at the same point on $|z|=1$, where $q(z)=z^{n} \overline{p(1 / \bar{z})}$. The result is best possible and equality holds for $p(z)=z^{n}+k^{n}$.

If we take $\lambda=1$ in Theorem 1 , then inequality (1.5) reduces to the following result, which is similar to inequality (1.3).

Corollary 1. If $p(z)$ is a polynomial of degree $n$, which does not vanish in $|z|<k, k<1$, then for $0<k<r<1$

$$
M(p, r) \geq \frac{r^{n}+k^{n}}{1+k^{n}} M(p, 1),
$$

provided $\left|p^{\prime}(z)\right|$ and $\left|q^{\prime}(z)\right|$ attain the maximum at the same point on $|z|=1$, where $q(z)=z^{n} \overline{p(1 / \bar{z})}$. The result is best possible and equality holds for $p(z)=z^{n}+k^{n}$.

Our next result further improves upon inequality (1.6). 
Theorem 2. If $p(z)$ is a polynomial of degree $n$, which does not vanish in $|z|<k, k<1$, then for $0<k<r<1$

$$
M(p, r) \geq\left(\frac{r^{n}+k^{n}}{1+k^{n}}\right) M(p, 1)+\left(\frac{1-r^{n}}{1+k^{n}}\right) m(p, k)
$$

provided $\left|p^{\prime}(z)\right|$ and $\left|q^{\prime}(z)\right|$ attain the maximum at the same point on $|z|=1$, where $q(z)=z^{n} \overline{p(1 / \bar{z})}$ and $m(p, k)=\min _{|z|=k}|p(z)|$. The result is best possible and equality holds for $p(z)=z^{n}+k^{n}$.

2. Lemma. For the proofs of the theorems we need the following lemma due to Govil [3].

Lemma. If $p(z)$ is a polynomial of degree $n$, which does not vanish in $|z|<k, k \leq 1$, then

$$
M\left(p^{\prime}, 1\right) \leq \frac{n}{1+k^{n}} M(p, 1),
$$

provided $\left|p^{\prime}(z)\right|$ and $\left|q^{\prime}(z)\right|$ attain the maximum at the same point on $|z|=1$, where $q(z)=z^{n} \overline{p(1 / \bar{z})}$.

\section{Proofs of the theorems.}

Proof of Theorem 1. If $p(z) \neq 0$ in $|z|<k, k<1$ and $0<t<1, k<t$, then $P(z)=p(t z)$ has no zero in $|z|<k / t, k / t<1$. Hence applying above Lemma to the polynomial $P(z)$, we get

$$
M\left(P^{\prime}, 1\right) \leq \frac{n}{1+k^{n} / t^{n}} M(P, 1)
$$

which is equivalent to

$$
M\left(p^{\prime}, t\right) \leq \frac{n t^{n-1}}{t^{n}+k^{n}} M(p, t) .
$$

For $0<r<\lambda \leq 1$ and $0<\theta \leq 2 \pi$, we have

$$
p\left(\lambda e^{i \theta}\right)-p\left(r e^{i \theta}\right)=\int_{r}^{\lambda} e^{i \theta} p^{\prime}\left(t e^{i \theta}\right) d t
$$

This implies

$$
\left|p\left(\lambda e^{i \theta}\right)-p\left(r e^{i \theta}\right)\right| \leq \int_{r}^{\lambda}\left|p^{\prime}\left(t e^{i \theta}\right)\right| d t
$$

which gives

$$
\left|p\left(\lambda e^{i \theta}\right)\right| \leq\left|p\left(r e^{i \theta}\right)\right|+\int_{r}^{\lambda}\left|p^{\prime}\left(t e^{i \theta}\right)\right| d t
$$

which further implies

$$
M(p, \lambda) \leq M(p, r)+\int_{r}^{\lambda} M\left(p^{\prime}, t\right) d t .
$$


Combining the above inequality with (3.1), we get

$$
M(p, \lambda) \leq M(p, r)+\int_{r}^{\lambda} \frac{n t^{n-1}}{t^{n}+k^{n}} M(p, t) d t .
$$

If we choose

$$
\phi(\lambda)=M(p, r)+\int_{r}^{\lambda} \frac{n t^{n-1}}{t^{n}+k^{n}} M(p, t) d t
$$

then

$$
\phi^{\prime}(\lambda)=\frac{n \lambda^{n-1}}{\lambda^{n}+k^{n}} M(p, \lambda)
$$

and inequality (3.2), gives

$$
\phi^{\prime}(\lambda)-\frac{n \lambda^{n-1}}{\lambda^{n}+k^{n}} \phi(\lambda) \leq 0 .
$$

Multiplying the above inequality by $\left(\lambda^{n}+k^{n}\right)^{-1}$, we get

$$
\frac{d}{d \lambda}\left\{\left(\lambda^{n}+k^{n}\right)^{-1} \phi(\lambda)\right\} \leq 0
$$

which implies that $\left(\lambda^{n}+k^{n}\right)^{-1} \phi(\lambda)$ is a non-increasing function of $\lambda$ in $(0,1)$. Therefore for $0<k<r<\lambda \leq 1$, we have

$$
\phi(r) \geq\left(\frac{r^{n}+k^{n}}{\lambda^{n}+k^{n}}\right) \phi(\lambda) .
$$

Now since $\phi(r)=M(p, r)$ and $\phi(\lambda) \geq M(p, \lambda)$, we get

$$
M(p, r) \geq\left(\frac{r^{n}+k^{n}}{\lambda^{n}+k^{n}}\right) M(p, \lambda) .
$$

Which completes the proof of Theorem 1 .

Proof of Theorem 2. If $p(z)$ is a polynomial of degree $n$ having no zero in $|z|<k, k<1$ and if $m(p, k)=\min _{|z|=k}|p(z)|$, then for every $\alpha$ with $|\alpha|<1$, the polynomial $p(z)-\alpha m(p, k)$ has no zero in $|z|<k, k<1$. This result is clear if $p(z)$ has a zero on $|z|=k$, for then $m(p, k)=0$ and therefore $p(z)-\alpha m(p, k)=p(z)$. In case $p(z)$ has no zero on $|z|=k$, then for every $\alpha$ with $|\alpha|<1$, we have $|p(z)|>|\alpha| m(p, k)$ on $|z|=k$ and on applying Rouche's theorem the result follows. Thus $p(z)-\alpha m(p, k)$ has no zero in $|z|<k, k<1$ and hence, applying inequality (1.6) to $p(z)-\alpha m(p, k)$, we get

$$
M(p-\alpha m(p, k), r) \geq\left(\frac{r^{n}+k^{n}}{1+k^{n}}\right) M(p-\alpha m(p, k), 1),
$$

which implies

$$
M(p-\alpha m(p, k), r) \geq\left(\frac{r^{n}+k^{n}}{1+k^{n}}\right)\{M(p, 1)-|\alpha| m(p, k)\} .
$$


Now choosing argument of $\alpha$ on left hand side of (3.3), we get

$$
M(p, r)-|\alpha| m(p, k) \geq\left(\frac{r^{n}+k^{n}}{1+k^{n}}\right)\{M(p, 1)-|\alpha| m(p, k)\},
$$

which is equivalent to

$$
M(p, r) \geq\left(\frac{r^{n}+k^{n}}{1+k^{n}}\right) M(p, 1)+\left(\frac{1-r^{n}}{1+k^{n}}\right)|\alpha| m(p, k)
$$

and letting $|\alpha| \rightarrow 1$, we get

$$
M(p, r) \geq\left(\frac{r^{n}+k^{n}}{1+k^{n}}\right) M(p, 1)+\left(\frac{1-r^{n}}{1+k^{n}}\right)|\alpha| m(p, k) .
$$

This completes the proof of Theorem 2 .

\section{REFERENCES}

[1] Ankeny, N. C., Rivlin, T. J., On a theorem of S. Bernstein, Pacific J. Math. 5 (1955), 849-852.

[2] Aziz, A., Growth of polynomials whose zeros are within or outside a circle, Bull. Austral. Math. Soc. 35 (1987), 247-256.

[3] Govil, N. K., On a theorem of S. Bernstein, Proc. Nat. Acad. Sci. India Sect. A 50 (1980), 50-52.

[4] Pólya, G., Szegö, G., Aufgaben und Lehrsatze aus der Analysis, Springer-Verlag, Berlin, 1925.

K. K. Dewan

Department of Mathematics

Faculty of Natural Sciences

Jamia Millia Islamia (Central University)

New Delhi-110025, India

e-mail: kkdewan123@yahoo.co.in

Received August 12, 2008
Sunil Hans

Department of Mathematics

Faculty of Natural Sciences

Jamia Millia Islamia (Central University)

New Delhi-110025, India

e-mail: sunil.hans82@yahoo.com 